\title{
Efectos del Tratamiento con Vitamina $E$ en el Tubo Neural y Médula Espinal en Embriones y Fetos de Ratones Mus musculus Expuestos al uso de Ácido Valproico
}

\author{
Effects of Treatment with Vitamin $E$ in the Neural Tube and Spinal Cord \\ Mus musculus Mouse Embryos Exposed to the use of Valproic Acid
}

\author{
Daniel Conei Valencia,*,,***; Bárbara Soler Guerra*; Gustavo Saint-Pierre Contreras*; \\ Jovita Ortiz Contreras ${ }^{* * * * *}$ \& Mariana Rojas Rauco*
}

CONEI, V. D.; SOLER, G. B., SAINT-PIERRE, C. G.; ORTIZ, C. J. \& ROJAS, R. M. Efectos del tratamiento con vitamina E en el tubo neural y médula espinal en embriones y fetos de ratones Mus musculus expuestos al uso de ácido valproico. Int. J. Morphol., 34(2):732-741, 2016.

RESUMEN: El ácido valproico (VPA) es el principal anticonvulsivante utilizado contra la epilepsia durante la gestación. Sin embargo, en etapas iniciales del embarazo actúa como teratógeno y ocasiona malformaciones como fisura labio-palatina, alteraciones en el desarrollo genital y espina bífida, siendo esta última la más frecuente. Esto se produce debido al aumento de especies reactivas de oxígeno, pudiendo contrarrestarse administrando vitamina E. El objetivo fue determinar si la vitamina E disminuye el daño en tubo neural y médula espinal de embriones y fetos de ratonas expuestas a VPA. Se conformaron 8 grupos de animales. A los 8 días postfecundación se les administró a los grupos 1 y 5 suero fisiológico $0,3 \mathrm{~mL}$; grupos 2 y 6 VPA 600 mg/Kg; grupos 3 y 7 VPA 600 mg/Kg y vitamina E 200 UI/Kg; grupos 4 y 8 vitamina E 200 UI/kg. A los 12 días post-fecundación, se sacrificaron los grupos 1, 2, 3 y 4, y a los 17 días los restantes grupos. Los embriones fueron procesados y teñidos con cresil violeta, observándose cortes histológicos a nivel cervical, torácico y lumbar. Los grupos tratados con vitamina E presentaron menor cantidad de neuroblastos y motoneuronas, pero de tamaño mayor en comparación al grupo tratado con VPA ( $\mathrm{p}<0,05)$, siendo similares a los grupos controles. Al comparar el tubo neural y médula espinal en los distintos niveles (cervical, torácico y lumbar), no hubo diferencias estadísticamente significativas. La administración prenatal de vitamina $\mathrm{E}$ disminuye los defectos en tubo neural y médula espinal de embriones de 12 y 17 días de gestación sometidos a VPA.

PALABRAS CLAVE: Ácido valproico; Vitamina E; Médula espinal; Tubo neural.

\section{INTRODUCCIÓN}

El uso de anticonvulsivantes es fundamental para el manejo de la epilepsia y trastornos del ánimo. Para el tratamiento de la epilepsia, los fármacos de primera línea son carbamazepina, fenitoína y ácido valproico (VPA), utilizándose como monoterapia el fármaco más adecuado a la dosis mínima capaz de controlar la patología (Ministerio de Salud, 2009).

Dentro de los fármacos mencionados, el VPA es uno de los más prescritos debido a su alta eficacia, ya que logra la inhibición de canales de sodio voltaje dependiente y del metabolismo neuronal, además de aumentar la neurotransmisión de GABA y reducir la neurotransmisión de aspartato (Morland et al., 2012).
Durante el embarazo, el uso de fármacos antiepilépticos conlleva a un importante riesgo para el desarrollo del embrión y feto, ya que se ha demostrado una relación de tipo causaefecto entre el uso de antiepilépticos y la incidencia de malformaciones congénitas, aumentando el riesgo de adquirirlas entre un 5 a $9 \%$ en comparación al 1 a $3 \%$ presente en gestaciones fisiológicas (Ornoy, 2009). Se estima que el ácido valproico es el más teratogénico, con un riesgo de causar malformaciones de un 9,3\% (Ahmed et al., 2014).

El daño potencial del VPA se puede separar en tres grandes grupos: 1) Anomalías congénitas mayores a nivel cardiaco, fisura labio-palatina, anomalías del tracto urina-

\footnotetext{
Laboratorio de Embriología Comparada, Programa de Anatomía y Biología del Desarrollo, Instituto de Ciencias Biomédicas, Facultad de Medicina, Universidad de Chile, Santiago, Chile.

** Centro de Investigación en Ciencias Biomédicas, Universidad Autónoma de Chile, Temuco, Chile.

*** Universidad Espíritu Santo, Guayaquil, Ecuador.

***** Departamento de Promoción de la Salud de la Mujer y el Recién Nacido, Facultad de Medicina, Universidad de Chile, Santiago, Chile.
} 
rio, de los miembros y del cerebro; 2) Síndrome fetal del ácido valproico, afectando principalmente al complejo cráneo-facial, con dismorfia facial, y otros órganos, como la genitalia externa y el tubo neural, siendo más frecuente la espina bífida entre el 1 a $2 \%$ de los casos; y 3) Desórdenes del desarrollo, afectando fundamentalmente la función cognitiva y el comportamiento, pudiendo incluso causar autismo (Ornoy; Go et al., 2012).

Es así como la Academia Americana de Neurología recomienda evitar el uso de este medicamento y reemplazarlo por otro de menor riesgo de malformación, como carbamazepina o fenitoína, cuando las condiciones de la paciente así lo permitan (Ahmed et al.). No obstante, aquellas gestantes que les sea imprescindible la ingesta de VPA para manejar adecuadamente su enfermedad, es recomendable no descontinuar su uso y contar con el asesoramiento médico adecuado para su manejo (Kälviäinen \& Tomson, 2006).

El efecto del medicamento sobre el tubo neural se relaciona con su uso en los 17 a 30 días post-concepcional, por ser un periodo crítico del desarrollo del sistema nervioso embrionario. Estos efectos son directamente proporcionales a las dosis utilizadas (Gaviria, 2008).

Dentro de las causas de las alteraciones del sistema nervioso asociadas al uso de VPA se encuentra el estrés oxidativo, el cual consiste en un desequilibrio entre la producción de especies reactivas del oxígeno (ROS) y/o una disminución en los antioxidantes enzimáticos y no enzimáticos (Dorado et al., 2003). Esto puede producir directamente daño en macromoléculas tales como DNA, proteínas y lípidos, alteración de las vías de señalización celular, a través de la activación de factores de transcripción sensibles a óxido reducción afectando todos los procesos celulares claves en la neurogénesis (Sha \& Winn, 2010).

Respecto a la detoxificación de las ROS, ésta puede alcanzarse a través de mecanismos antioxidantes enzimáticos y no enzimáticos (Rajeshwari et al., 2014). Pese a que el embrión está equipado con mecanismos defensivos antioxidantes enzimáticos, diversos estudios han demostrado que la expresión de superóxido dismutasa, catalasa y glutatión peroxidasa como enzimas detoxificantes, son más bajos durante el desarrollo embrionario temprano comparado con los niveles maternos, por tanto, no resultan suficientes para revertir los efectos tóxicos de las ROS (Kase et al., 2012).

Se sabe además que el uso crónico de VPA disminuye los niveles de vitamina $\mathrm{E}$ y glutatión peroxidasa
(Zhang et al, 2010). Estudios en modelo murino han demostrado que los efectos generales del ácido valproico podrían ser revertidos con la administración previa de antioxidantes como la vitamina $\mathrm{C}$ o la vitamina $\mathrm{E}$, siendo esta última capaz de traspasar la barrera uteroplacentaria mediante transporte activo. Su acción principal es prevenir la oxidación de los lípidos de la membrana celular, ya que neutraliza la acción desestabilizadora de los radicales libres del oxígeno sobre dicha estructura (Al Deeb et al., 2000).

Adicionalmente, se ha observado que la administración conjunta de ácido fólico y vitamina $\mathrm{E}$ disminuye los efectos deletéreos del ácido valproico a nivel del nervio isquiático (Aluclu et al., 2009). Sin embargo, no se reportan estudios experimentales que determinen dichos beneficios en el sistema nervioso central propiamente tal.

En este contexto, el propósito de este estudio es determinar las diferencias histológicas en el tubo neural y médula espinal en embriones y fetos de ratonas expuestos a terapia antiepiléptica con ácido valproico, expuestos a esta terapia en conjunto con vitamina $\mathrm{E}$ y expuestos sólo a vitamina E de 12 y 17 días de gestación.

\section{MATERIAL Y MÉTODO}

Se utilizaron 40 ratonas adultas jóvenes (Mus musculus), las que se dividieron en 8 grupos de 5 ratonas gestantes cada uno (ver Tabla I). Éstas se colocaron en jaulas con machos reproductivamente sanos y jóvenes. Se consideró como el día 1 de gestación el momento en que se verifica la presencia de tapón mucoso a nivel vaginal, lo que significa que hubo cópula pero no asegura que hubo fecundación.

A los 8 días post coital (p.c.) se procedió a dividir a las hembras gestantes en 8 grupos y recibieron los siguientes tratamientos vía oral en micropipetas de $200 \mu \mathrm{L}$ :

Los grupos 1 y 5 fueron tratados con $0,3 \mathrm{~mL}$ de suero fisiológico, conformando los grupos control ( $\mathrm{NaCl} \mathrm{0,9 \% ).}$

Los grupos 2 y 6 recibieron $600 \mathrm{mg} / \mathrm{Kg}$ de ácido valproico (Atemperator ${ }^{\circledR}$, Laboratorio Recalcine ${ }^{\circledR}$ ).

A los grupos 3 y 7 se les administró 600 mg/Kg de ácido valproico y $200 \mathrm{UI} / \mathrm{Kg}$ de vitamina E (Laboratorio Fasa $^{\circledR}$ ).

A los grupos 4 y 8 se les suministró 200 UI/Kg de vitamina $\mathrm{E}$. 
Tabla I. Esquema de intervención.

\begin{tabular}{llll}
\hline Grupos & $\begin{array}{l}\text { Días de } \\
\text { Gestación }\end{array}$ & Medicamento & Dosis \\
\hline 1 & 12 & Suero Fisiológico & $0,3 \mathrm{~mL}$ \\
2 & 12 & VPA & $600 \mathrm{mg} / \mathrm{Kg}$ \\
3 & 12 & VPA + Vitamina E & $600 \mathrm{mg} / \mathrm{Kg}+200 \mathrm{UI} / \mathrm{Kg}$ \\
4 & 12 & Vitamina E & $200 \mathrm{UI} / \mathrm{Kg}$ \\
5 & 17 & Suero Fisiológico & $0,3 \mathrm{~mL}$ \\
6 & 17 & VPA & $600 \mathrm{mg} / \mathrm{Kg}$ \\
7 & 17 & VPA + Vitamina E & $600 \mathrm{mg} / \mathrm{Kg}+200 \mathrm{UI} / \mathrm{Kg}$ \\
8 & 17 & Vitamina E & $200 \mathrm{UI} / \mathrm{Kg}$ \\
\hline
\end{tabular}

\section{RESULTADOS}

Embriones de 12 días de gestación (grupos $1,2,3$ y 4). Cantidad de neuroblastos en la placa basal del tubo neural: La cantidad de neuroblastos presentes en el tubo neural de los embriones de los 4 grupos estudiados se presenta en la Figura 1, donde se observa una mayor cantidad de células nerviosas en el grupo tratado con VPA $(24,47 \pm 2,065 ; \mathrm{p}<0,0001)$. Por otro lado, el grupo control fue el que obtuvo una menor cantidad de neuroblastos en el tubo neural $(13,2 \pm 1,473$;

A los 12 días de gestación las ratonas de los grupos $1,2,3$ y 4 se anestesiaron y eutanasiaron con solución de eutanasia xilacina en dosis 1,1 a 2,2 mg/Kg y ketamina en dosis de 10 a $20 \mathrm{mg} / \mathrm{Kg}$. Posteriormente, con la misma metódica se eutanasiaron las ratonas de los grupos 5, 6, 7 y 8 correspondiente a los grupos de 17 días de gestación (ver Tabla I). El presente estudio contó con la aprobación del Comité de Bioética sobre Investigación en Animales de la Facultad de Medicina de la Universidad de Chile.

Los embriones y fetos de cada camada que fueron fijados en solución Bouin y procesados según técnica histológica. Se cortaron a $5 \mu \mathrm{m}$ en forma transversal a nivel de la columna cervical, torácico y lumbar, en un micrómetro Microm HM350R y se montaron en portaobjetos xilanizados.

Se empleó la técnica de Cresil Violeta (Certistain ${ }^{\circledR}$, Merck, 2011). Se procedió a desparafinar los cortes de manera habitual y rehidratar, teñir en solución de violeta de cresilo durante $20 \mathrm{~min}$, sumergir brevemente en etanol 70 $\%$, enjuagar en etanol $96 \%$, aclarar en xileno y montar con Entellan ${ }^{\circledR}$ durante 2 min en cada caso.

Los cortes histológicos fueron fotografiados en un microscopio Zeiss con cámara fotográfica Cannon Power Shot A640 conectada a un computador. Se contabilizaron y midieron los neuroblastos presentes en la región basal del tubo neural en embriones de 12 días de gestación. En el caso de los fetos de 17 días de gestación, se cuantificaron y midieron las neuronas motoras presentes en el asta ventral de la médula espinal. En ambos casos se utilizó el método disector con Stepanizer, y se midieron con el programa Axiovision 6.0. También se realizó una descripción morfológica de carácter cualitativo de las células nerviosas.

Los resultados se presentaron en promedio y desviación estándar. Para la comparación de los grupos se utilizó test de ANOVA para medidas anidas. Se consideró una diferencia significativa cuando el valor de $\mathrm{p}$ fue menor a 0,05. Se utilizó el programa GraphPad Prism 6. $\mathrm{p}<0,0001$ ), siendo una diferencia estadísticamente significativa. Entre el grupo que se le administró VPA y aquel que recibió VPA en forma conjunta con vitamina $\mathrm{E}$, hubo diferencias significativas, presentando este último un valor de 14,8 $\pm 1,698$ ( $\mathrm{p}<0,0001)$ neuroblastos. Al contrastar los resultados obtenidos entre el grupo tratado con VPA y el que recibió vitamina E, también se observó diferencias significativas $(16,3 \pm 2,794$ neuroblastos; $\mathrm{p}<0,0001)$.

No hubo diferencias estadísticamente significativas entre los grupos control y los que recibieron VPA en forma conjunta con vitamina $\mathrm{E}$ y vitamina $\mathrm{E}$ sola. No obstante, los 4 grupos se comportaron de forma similar en los distintos segmentos del tubo neural analizados (cervical, torácico y lumbar), sin obtenerse resultados significativos.

Tamaño de neuroblastos en la placa basal del tubo neural: Entre los 4 grupos estudiados, el tamaño de los neuroblastos presentes en el tubo neural (Fig. 2), fue menor en el grupo tratado con VPA $(6,393 \pm 0,135 \mu \mathrm{m}$; $\mathrm{p}<0,0001)$. Al compararlo con el grupo control, se evidenció un mayor tamaño de neuroblastos $(7,268 \pm 0,123$ $\mu \mathrm{m} ; \mathrm{p}<0,0001)$, siendo una diferencia estadísticamente significativa. Entre el grupo que se le administró VPA y el que recibió VPA en forma conjunta con vitamina $\mathrm{E}$, hubo diferencias significativas, presentando este último un tamaño de neuroblastos de 7,801 $\pm 0,215 \mu \mathrm{m}$ ( $<<0,0001)$. Por último, entre el grupo tratado con VPA y el que recibió vitamina $\mathrm{E}$, también se observaron diferencias significativas $(7,612 \pm 0,121 \mu \mathrm{m} ; \mathrm{p}<0,0001)$.

$\mathrm{Al}$ igual que en la variable número de neuroblastos, no hubo diferencias estadísticamente significativas entre los grupos control y los que recibieron VPA en forma conjunta con vitamina $\mathrm{E}$ y vitamina $\mathrm{E}$ sola. A su vez, los 4 grupos se comportaron de forma similar en los distintos segmentos del tubo neural analizados (cervical, torácico y lumbar), sin obtenerse resultados significativos. 


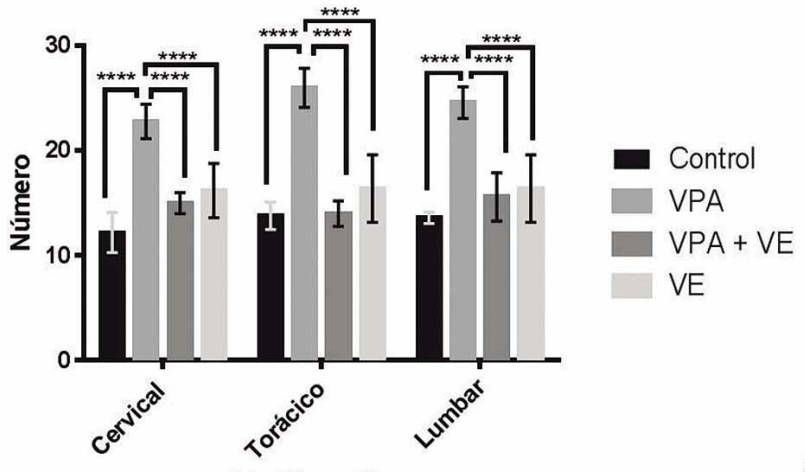

Fig. 1. Comparación del número de neuroblastos en la placa basal del tubo neural entre grupo 1 (Control), grupo 2 (VPA), grupo 3 (VPA + VE) y grupo 4 (VE), según el nivel analizado (cervical, torácico y lumbar). Graficado desviación estándar (DE). **** Valor de $\mathrm{p}<0,0001$.

Forma de neuroblastos en la placa basal del tubo neural: En todos los grupos los neuroblastos presentaron forma de pera, núcleos ovoides y citoplasma definido. En cuanto a la sustancia cromófila (sustancia de Nissl), el grupo control fue el que presentó una marcada tinción. Estos hallazgos se presentaron en todos los niveles analizados (Figs. 3, 4, 5 y 6).

Embriones de 17 días de gestación (grupos 5, 6, 7 y 8). Cantidad de motoneuronas en el asta ventral de la médula espinal: En el Figura 7 se muestra la cantidad de motoneuronas presentes en la médula espinal de los embriones de los 4 grupos restantes estudiados. Se observó una mayor cantidad de células nerviosas en el grupo tratado con VPA $(17,13 \pm 1,245 ; \mathrm{p}<0,01)$. El grupo que se le administró

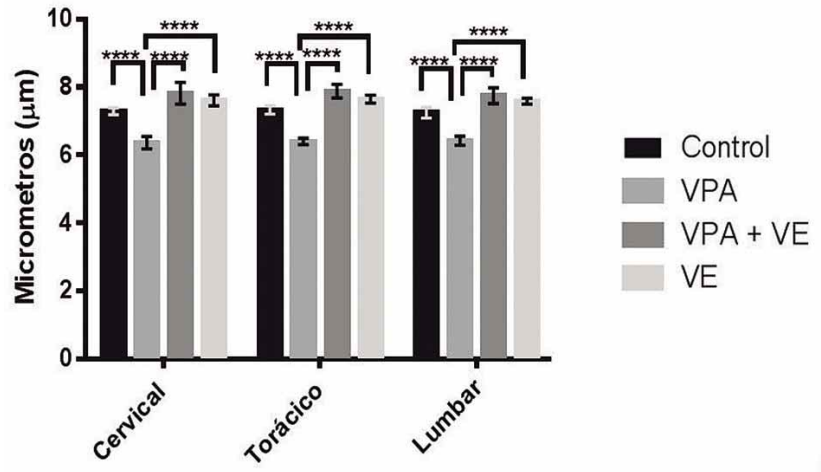

Fig. 2. Comparación del tamaño de neuroblastos $(\mu \mathrm{m})$ en la placa basal del tubo neural entre grupo 1 (Control), grupo 2 (VPA), grupo 3 (VPA + VE) y grupo 4 (VE), según el nivel analizado (cervical, torácico y lumbar). Graficado desviación estándar (DE). $* * * *$ Valor de $\mathrm{p}<0,0001$.

VPA de manera conjunta con vitamina $\mathrm{E}$ fue el que obtuvo una menor cantidad de neuronas motoras en el asta ventral de la médula espinal $(12,53 \pm 1,597 ; \mathrm{p}<0,05)$, y se obtuvo una diferencia estadísticamente significativa al comparar dichos grupos, aumentando gradualmente según el nivel de la médula espinal analizada (nivel cervical $\mathrm{p}<0,05$; nivel torácico $\mathrm{p}<0,01$; nivel lumbar $\mathrm{p}<0,001)$. Entre el grupo que se le administró VPA y el grupo control, hubo diferencias significativas, presentando este último un valor de $12,86 \pm 1,884$ motoneuronas, con valor de $\mathrm{p}<0,01$ en todos los niveles estudiados. Por último, el grupo tratado con VPA y el que recibió vitamina $\mathrm{E}$, tuvo diferencias significativas, obteniéndose un valor de $\mathrm{p}<0,001$ a nivel cervical, $\mathrm{p}<0,05$ a nivel torácico y $\mathrm{p}<0,01$ a nivel lumbar $(13,6 \pm 1,447$ neuronas motoras).

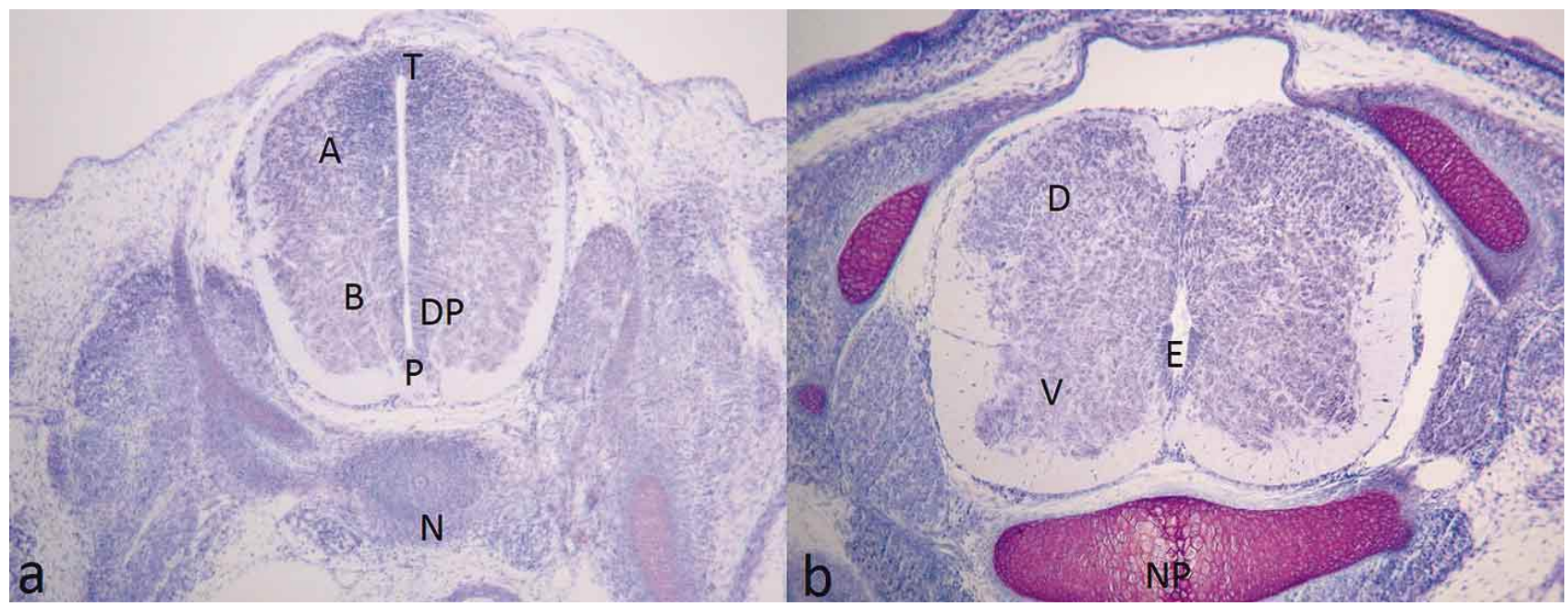

Fig. 3. a) Corte histológico de tubo neural de embrión de ratón de 12 días de gestación de grupo control a nivel torácico teñido con violeta de cresilo. 100x. (T) placa del techo, (A) placa alar, (B) placa basal), (DP) dominio progenitor, (P) placa del piso, (N) notocorda. b). Corte histológico de médula espinal de feto de ratón de 17 días de gestación de grupo control a nivel torácico teñido con violeta de cresilo. 100X. (D) asta dorsal, (V) asta ventral, (E) epéndimo, (NP) núcleo pulposo. 


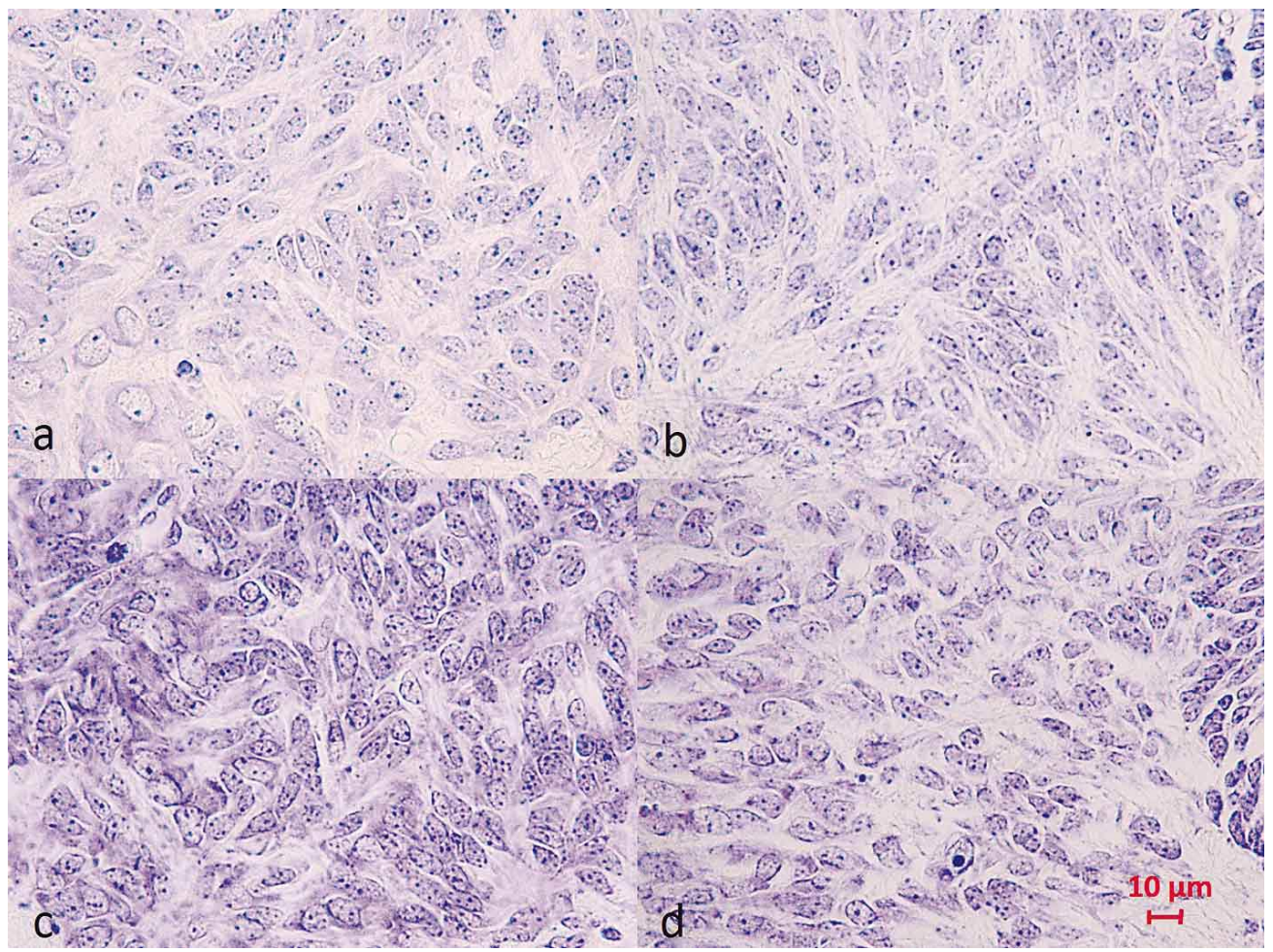

Fig. 4. Cortes histológicos de la placa basal del tubo neural de embriones de ratón de 12 días de gestación a nivel cervical teñido con violeta de cresilo al 400X. a) Grupo 1 (Control). b) Grupo 2 (VPA). c) Grupo 3 (VPA + VE). d) Grupo 4 (VE).

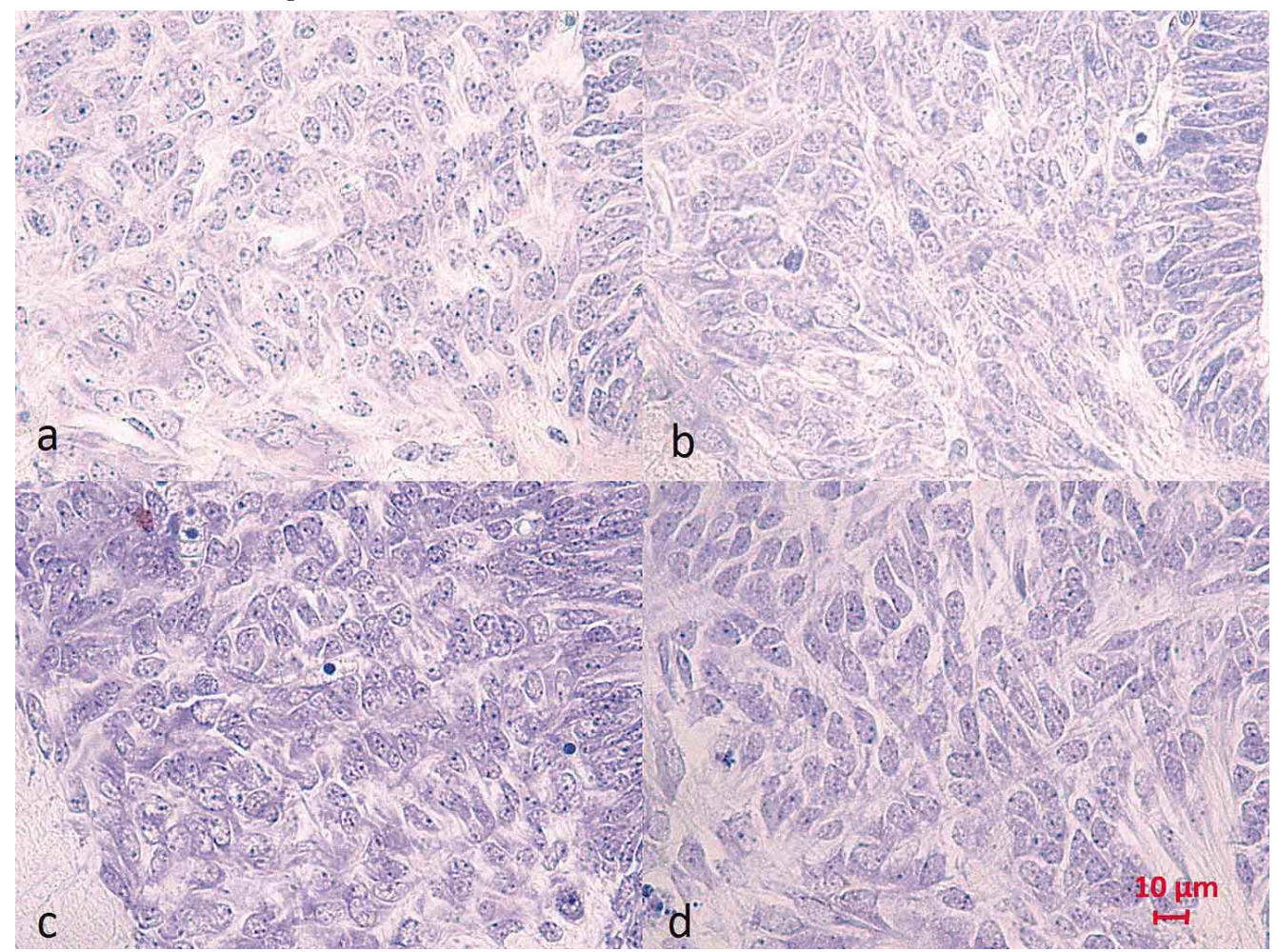

Fig. 5. Cortes histológicos de la placa basal del tubo neural de embriones de ratón de 12 días de gestación a nivel torácico teñido con violeta de cresilo al 400X. a) Grupo 1 (Control). b) Grupo 2 (VPA). c) Grupo $3(\mathrm{VPA}+\mathrm{VE})$. d) Grupo 4 (VE). 


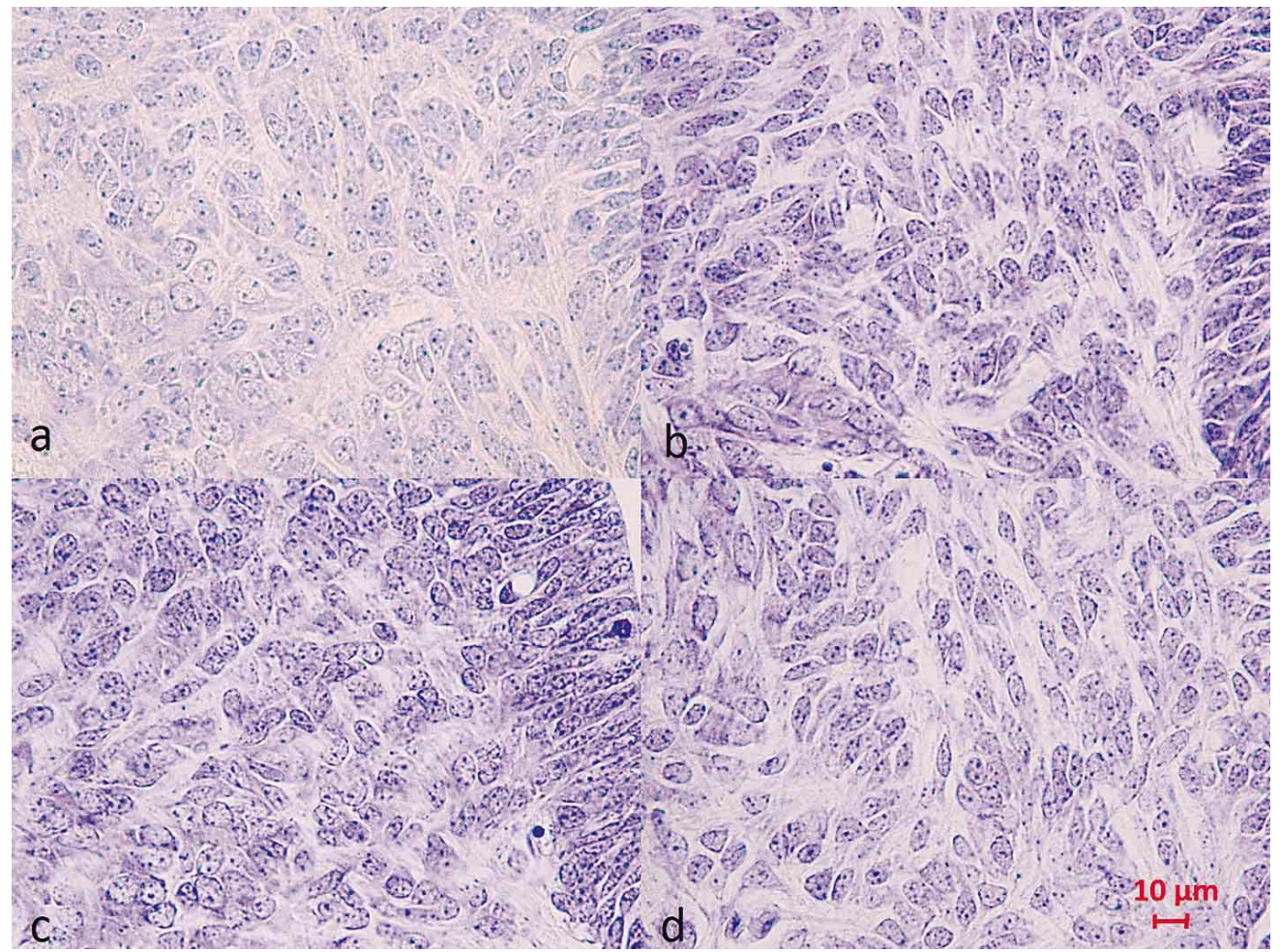

Fig. 6. Cortes histológicos de la placa basal del tubo neural de embriones de ratón de 12 días de gestación a nivel lumbar teñido con violeta de cresilo al 400X. a) Grupo 1 (Control). b) Grupo 2 (VPA). c) Grupo 3 $(\mathrm{VPA}+\mathrm{VE})$. d) Grupo 4 (VE).

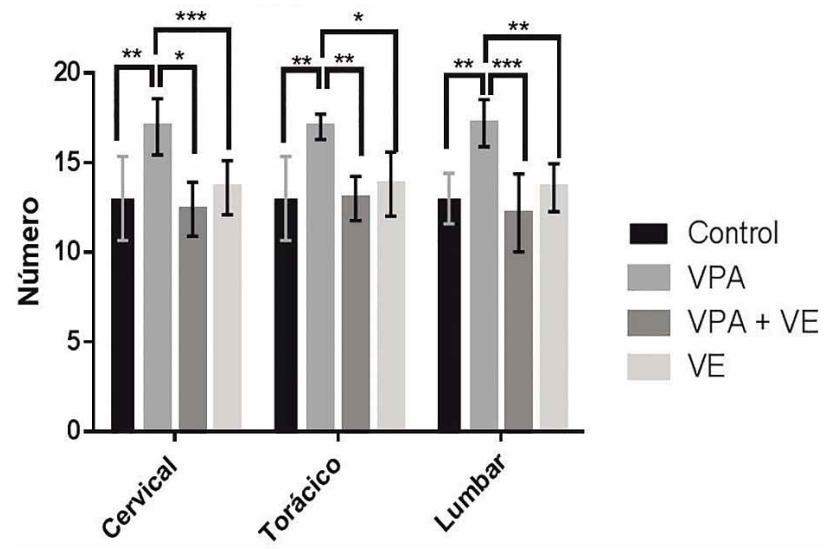

Fig. 7. Comparación del número de motoneuronas en el asta ventral de la médula espinal entre grupo 5 (Control), grupo 6 (VPA), grupo 7 (VPA + VE) y grupo 8 (VE), según el nivel analizado (cervical, torácico y lumbar). Graficado desviación estándar (DE). *Valor de $\mathrm{p}<0,05, * *$ valor de $\mathrm{p}<0,01 \mathrm{y} * * *$ valor de $\mathrm{p}<0,001$.

Al comparar los grupos control y los que recibieron VPA en forma conjunta con vitamina $\mathrm{E}$ y vitamina $\mathrm{E}$ sola, no se observó diferencias significativas.
Tamaño de motoneuronas en el asta ventral de la médula espinal: En cuanto al tamaño de los neuronas motoras presentes (Fig. 8) en los distintos grupos estudiados, se evidenció la presencia de células nerviosas de menor tamaño en el grupo tratado con VPA $(7,482 \pm 0,196 \mu \mathrm{m}$; $\mathrm{p}<0,0001$ ), en contraste con el grupo control, el cual presentó motoneuronas de mayor tamaño $(8,546 \pm 0,085 \mu \mathrm{m}$; $\mathrm{p}<0,0001$ ), obteniéndose una diferencia estadísticamente significativa. Al comparar el grupo que se le administró VPA y el que recibió VPA en forma conjunta con vitamina E, se observó en este último un tamaño de 8,675 $\pm 0,147$ $\mu \mathrm{m}$, con significancia estadística. Finalmente, se encontraron diferencias significativas al comparar el grupo tratado con VPA y el que recibió vitamina E (tamaño de motoneuronas de 8,806 $\pm 0,137 \mu \mathrm{m} ; \mathrm{p}<0,0001$ ).

No hubo diferencias estadísticamente significativas entre los grupos control y los que recibieron VPA en forma conjunta con vitamina $\mathrm{E}$ y vitamina $\mathrm{E}$ sola. A diferencia de la variable número de motoneuronas, hubo un comportamiento similar entre los 4 grupos en los distintos segmentos de la médula espinal analizados (cervical, torácico y lumbar), sin obtenerse resultados significativos. 


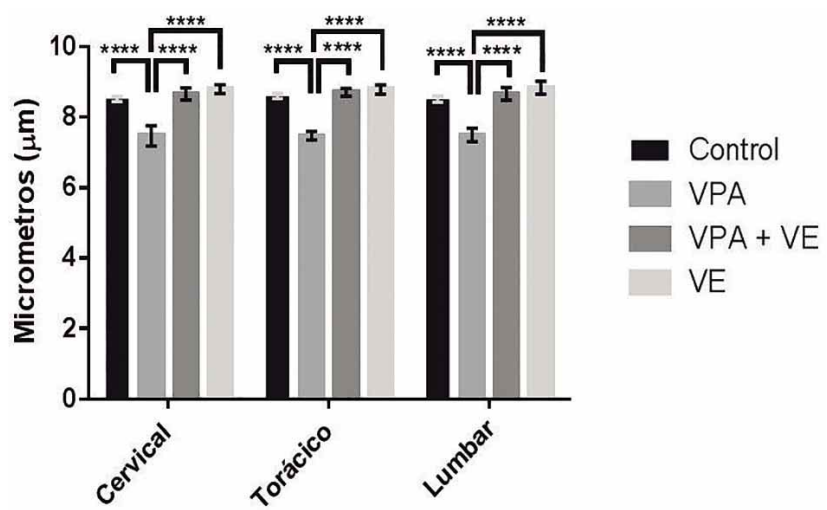

Fig. 8. Comparación del tamaño de motonueronas $(\mu \mathrm{m})$ en el asta ventral de la médula espinal entre grupo 5 (Control), grupo 6(VPA), grupo 7 (VPA + VE) y grupo 8 (VE), según el nivel analizado (cervical, torácico y lumbar). Graficado desviación estándar (DE). $* * * *$ Valor de $\mathrm{p}<0,0001$.

Forma de motoneuronas en el asta ventral de la médula espinal: Los 4 grupos presentaron motoneuronas de forma ovoide, de contornos definidos. Sin embargo, el grupo tratado con VPA a diferencia de otros grupos posee mayor pigmentación de la sustancia cromófila, células nerviosas agrupadas en ciertas zonas del asta ventral y menor definición de los nucléolos. Este patrón se presenta en todos los segmentos analizados (Figs. 3, 9, 10 y 11).

\section{DISCUSIÓN}

La presente investigación confirmó la hipótesis de que la vitamina $\mathrm{E}$ tiene un efecto beneficioso en el desarrollo del tubo neural y médula espinal en embriones y fetos de ratón tratados con VPA.

Los resultados permiten afirmar que la administración de ácido valproico induce un aumento en el número de células nerviosas tanto en los embriones de 12 días de gestación como en los fetos de 17 días de gestación. Esto concuerda con un estudio similar realizado en ratas, en donde la exposición prenatal a VPA produjo un incremento en el número de células progenitoras neurales (Go et al.). Un comportamiento similar ocurrió en este tipo de células, pero en cerebro posterior embrionario de peces cebra (Dozawa et al., 2014). Otra investigación realizada en ratas también arrojó un aumento de neuronas neocorticales causado por el mismo fármaco (Sabers et al., 2014).

Wang et al. (2015) explican este efecto mediante la vía de señalización de WNT. Este factor de crecimiento está involucrado en el control del crecimiento y diferenciación celular en el desarrollo del sistema nervioso central en modelos animales. En este experimento, se analizó la diferenciación neuronal de células progenitoras neurales

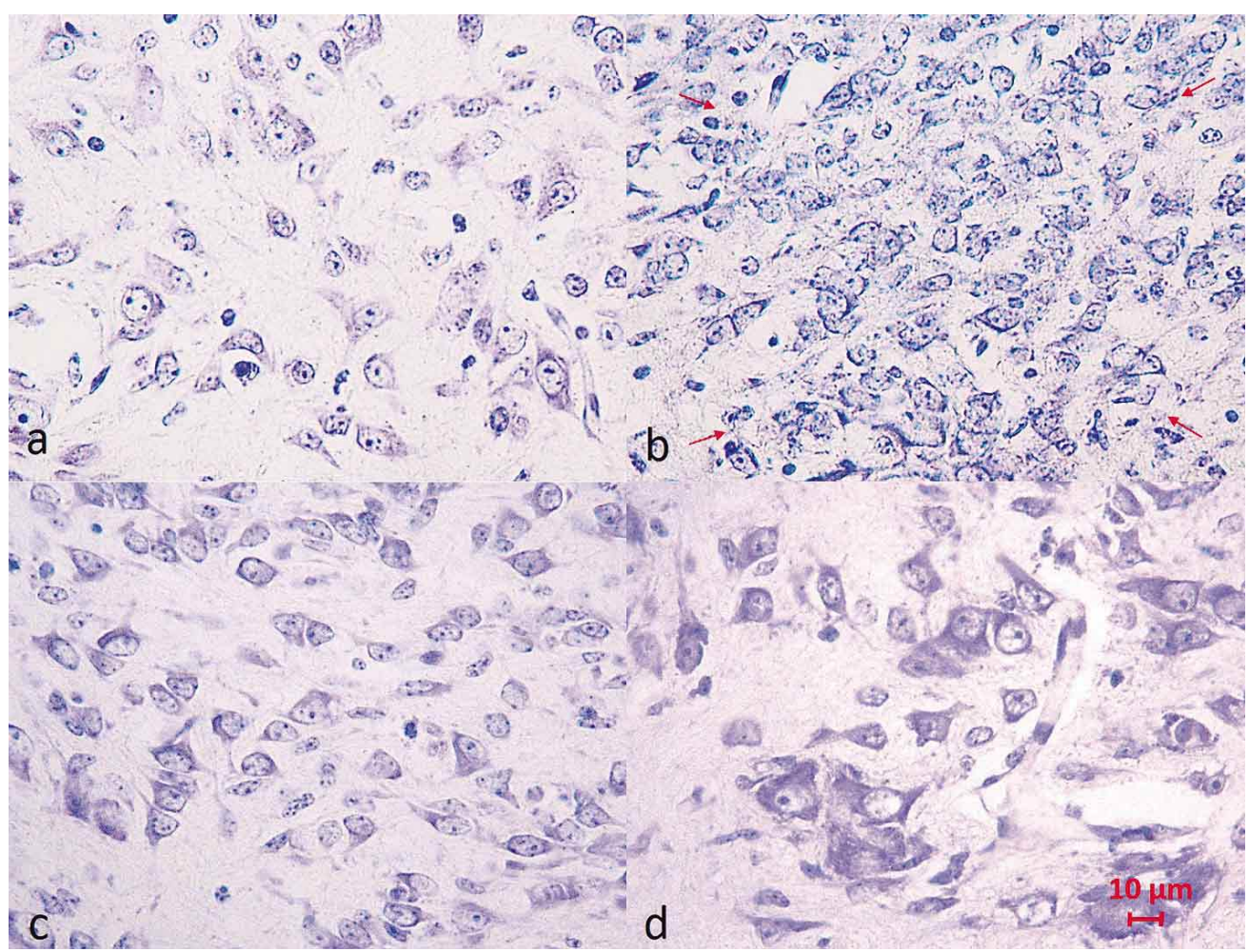

738
Fig. 9. Cortes histológicos del asta ventral de la médula espinal de ratón de 17 días de gestación a nivel cervical teñido con violeta de cresilo al 400X. a) Grupo 5 (Control). b) Grupo 6 (VPA). c) Grupo 7 (VPA + VE). d) Grupo 8 (VE). Flechas indican agrupación de motoneuronas en el centro del asta ventral de la médula espinal. 


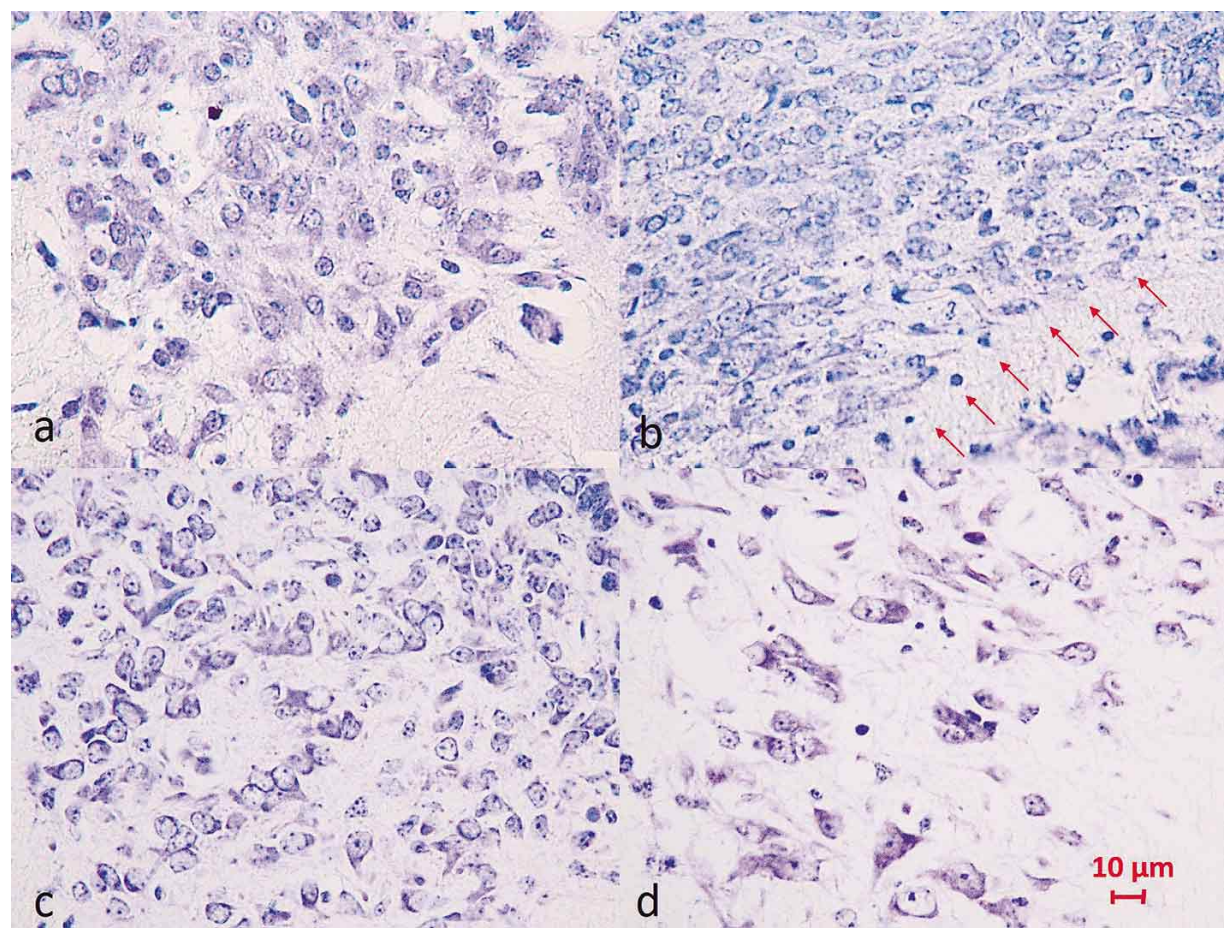

Fig. 10. Cortes histológicos del asta ventral de la médula espinal de ratón de 17 días de gestación a nivel torácico teñido con violeta de cresilo al 400X. a) Grupo 5 (Control). b) Grupo 6 (VPA). c) Grupo 7 (VPA + VE). d) Grupo 8 (VE). Flechas indican agrupación de motoneuronas hacia la derecha del asta ventral de la médula espinal.

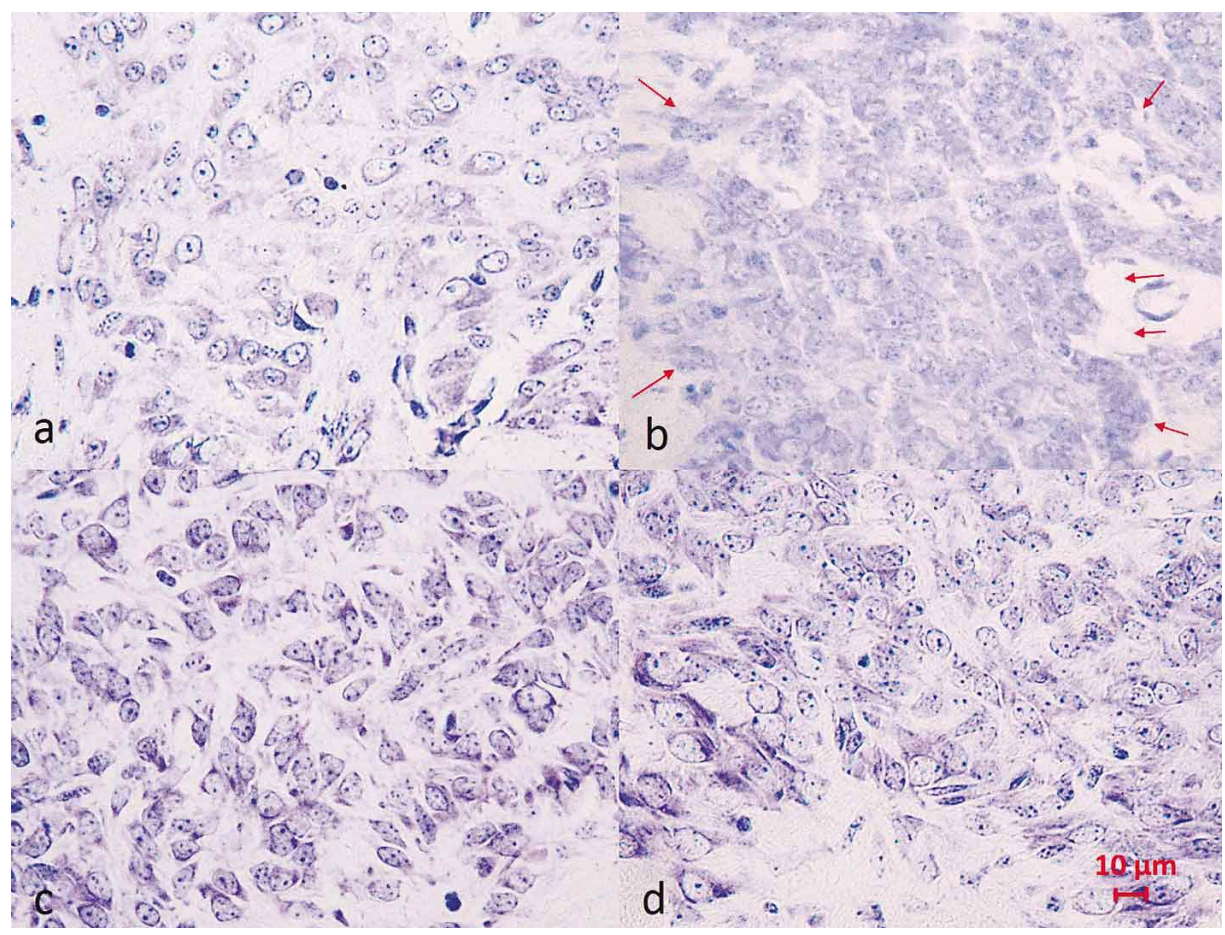

Fig. 11. Cortes histológicos del asta ventral de la médula espinal de ratón de 17 días de gestación a nivel torácico teñido con violeta de cresilo al 400X. a) Grupo 5 (Control). b) Grupo 6 (VPA). c) Grupo 7 (VPA + VE). d) Grupo 8 (VE). Flechas indican agrupación de motoneuronas en el centro del asta ventral de la médula espinal. 
inducida por medios de cultivo de VPA utilizando ensayo de inmunocitoquímica in vitro. Se observó una expresión de WNT-3a y $\beta$-catenina significativamente mayor en las células tratadas con VPA en comparación con los medios de control. Complementando esta explicación, un mecanismo que ha propuesto Tsai et al. (2008) es que la administración de VPA aumenta los niveles de factores antiapoptóticos como BCL-2 y BCL-X en las neuronas de la médula espinal, además de inducir la neurogénesis y promover la proliferación de astrocitos en ratas.

El efecto anti-apoptótico producido por el ácido valproico se debe a que al aumentar el estrés oxidativo, se inhibe la liberación de Citocromo C y con ello reduce la apoptosis de células. Esto se evidenció en un estudio elaborado por Lee et al. (2014), quienes administraron VPA a ratas con lesión en la médula espinal y observaron que este fármaco reduce la muerte celular de las neuronas motoras.

En el caso de la vitamina E, se pudo constatar que ninguno de los embriones o fetos tratados con este fármaco presentó malformaciones del tubo neural. Por lo tanto, es posible aseverar que la administración de vitamina $\mathrm{E}$ no produce defectos en las crías. Igualmente, se puede afirmar que los embriones que fueron tratados con ácido valproico y vitamina $E$ presentaron histología normal en comparación al grupo tratado con ácido valproico sólo.

En concordancia con esta investigación, existe un estudio desarrollado por Al Deeb et al., donde se demostró que la vitamina $\mathrm{E}$ disminuye los defectos en el tubo neural en ratones que se les administró VPA. Esto se ex- plica ya que la vitamina $\mathrm{E}$ en el tubo neural actúa como potente antioxidante, disminuyendo el estrés oxidativo suprimiendo la vía ROS, de esta forma regula la liberación de Citocromo $\mathrm{C}$ en el citosol, actuando como factor protector para el desarrollo del sistema nervioso (Yanamala et al., 2014).

Referente al tamaño de los neuroblastos y neuronas motoras, hubo una marcada diferencia entre los grupos que recibieron únicamente VPA en comparación con los demás. Si bien, no hay evidencia disponible que avale esta situación a nivel de tejido nervioso, sí se ha visto que en la glándula submandibular, músculos linguales y cartílago mandibular en fetos de ratón tratados con VPA, existe una diferenciación retardada, células de menor tamaño, núcleos pequeños y estructuras desorganizadas (Siéssere et al., 2004). Esto concuerda con lo reportado por un estudio similar relacionado a los efectos del ácido valproico sobre la morfología de órganos maternos y fetales, el cual arrojó cambios importantes a nivel histológico en órganos fetales en cuanto la forma de las células nerviosas, siendo el grupo tratado con VPA el que presentó menor tamaño celular (Emmanouil-Nikoloussi et al., 2004).

De acuerdo a lo anteriormente mencionado, este estudio concluye que al estudiar los efectos que produce la vitamina $E$ en tubo neural y médula espinal de embriones y fetos de ratón tratados con ácido valproico, se evidenció un rol protector sobre los efectos que produce el anticonvulsivante, ya que produjo células nerviosas de mayor tamaño y un número de neuroblastos y motoneuronas similar al grupo control.

CONEI, V. D.; SOLER, G. B., SAINT-PIERRE, C. G.; ORTIZ, C. J. \& ROJAS, R. M. Effects of treatment with vitamin E in the neural tube and spinal cord Mus musculus mouse embryos exposed to the use of valproic acid. Int. J. Morphol., 34(2):732-741, 2016.

SUMMARY: Valproic Acid (VPA) is the main anticonvulsant used for epilepsy throughout the gestation period. However, when used at early stages of pregnancy, it acts as a tetarogenic agent, causing congenital malformations such as cleft-lip and/or cleft palate, abnormal genital development and spina bifida, being the latter the most frequent. This is the result of the increase of reactive oxygen species, which can be countered with the supplementation of vitamin E. The aim was determine if vitamin E minimizes the damage to the neural tube and spinal cord of mice embryos and fetuses previously exposed to VPA. Eight groups of mice were constituted. Eight days post fertilization, groups 1 and 5 were administered $0,3 \mathrm{ml}$ of saline solution; groups 2 and $6600 \mathrm{mg} / \mathrm{Kg}$ of VPA, groups 3 and $7600 \mathrm{mg} / \mathrm{Kg}$ of VPA and 200UI/Kg of Vitamin E; groups 4 and $8200 \mathrm{UI} / \mathrm{Kg}$ of Vitamin E. 12 days after fertilization, groups 1, 2, 3 and 4 were euthanized, whereas in the case of the remaining groups, the same process was performed 17 days after fertilization. The embryos were stained with cresyl violet, thus enabling the observation of histological sections at cervical, thoracic and lumbar levels. Groups supplied with vitamin E presented a lower amount of neuroblasts and motoneurons. However, these elements were bigger in size compared to the group treated with VPA ( $<<0,05)$, being these results similar to those obtained with the control groups. When comparing the neural tube and spinal cord at different levels (cervical, thoracic and lumbar), no statistically significant differences were found. It was determined that prenatal administration of vitamin E lessens the damage to the neural tube and spinal cord of mice embryos of 12 and 17 days of gestation previously exposed to VPA 


\section{REFERENCIAS BIBLIOGRÁFICAS}

Ahmed, R.; Apen, K. \& Endean, C. Epilepsy in pregnancy - a collaborative team effort of obstetricians, neurologists and primary care physicians for a successful outcome. Aust. Fam. Physician, 43(3):112-6, 2014.

Al Deeb, S.; Al Moutaery, K.; Arshaduddin, M. \& Tariq, M. Vitamin E decreases valproic acid induced neural tube defects in mice. Neurosci. Lett., 292(3):179-82, 2000.

Aluclu, M.; Tuncer, M.; Guzel, A.; Aluclu, M. \& Akkus, M. The effects of valproic acid on sciatic nerve of fetal rats and protective effects of folic acid and vitamin E. Int. J. Morphol., 27(2):285-94, 2009.

Dorado, C.; Rugerio, C. \& Rivas, S. Estrés oxidativo y neurodegeneración. Rev. Fac. Med. UNAM, 46(6):229-35, 2003.

Dozawa, M.; Kono, H.; Sato, Y.; Ito, Y.; Tanaka, H. \& Ohshima, T. Valproic acid, a histone deacetylase inhibitor, regulates cell proliferation in the adult zebrafish optic tectum. Dev. Dyn., 243(11):1401-15, 2014.

Emmanouil-Nikoloussi, E. N.; Foroglou, N. G.; Kerameos-Foroglou, C. H. \& Thliveris, J. A. Effect of valproic acid on fetal and maternal organs in the mouse: a morphological study. Morphologie, 88(280):41-5, 2004.

Gaviria, S. Tratamiento del trastorno afectivo bipolar en el embarazo. Rev. Chil. Neuro-psiquiatr., 46(1):43-54, 2008

Go, H. S.; Kim, K. C.; Choi, C. S.; Jeon, S. J.; Kwon, K. J.; Han, S. H.; Lee, J.; Cheong, J. H.; Ryu, J. H.; Kim, C. H.; Ko, K. H. \& Shin, C. Y. Prenatal exposure to valproic acid increases the neural progenitor cell pool and induces macrocephaly in rat brain via a mechanism involving the GSK-3b/b-catenin pathway. Neuropharmacology, 63(6):1028-41, 2012.

Kälviäinen, R. \& Tomson, T. Optimizing treatment of epilepsy during pregnancy. Neurology, 67(12 Suppl. 4):S59-63, 2006.

Kase, B. A.; Northrup, H.; Morrison, A. C.; Davidson, C. M.; Goiffon, A. M.; Fletcher, J. M.; Ostermaier, K. K.; Tyerman, G. H. \& Au, K. S. Association of copper-zinc superoxide dismutase (SOD1) and manganese superoxide dismutase (SOD2) genes with nonsyndromic myelomeningocele. Birth Defects Res. A Clin. Mol. Teratol., 94(10):762-9, 2012.

Lee, J. Y.; Maeng, S.; Kang, S. R.; Choi, H. Y.; Oh, T. H.; Ju, B. G. \& Yune, T. Y. Valproic acid protects motor neuron death by inhibiting oxidative stress and endoplasmic reticulum stress-mediated cytochrome C release after spinal cord injury. J. Neurotrauma., 31(6):582-94, 2014.

Ministerio de Salud (MINSAL). Guía clínica: Epilepsia en el Adulto. Santiago de Chile, Ministerio de Salud (MINSAL), 2009.

Morland, C.; Nordengen, K. \& Gundersen, V. Valproate causes reduction of the excitatory amino acid aspartate in nerve terminals. Neurosci. Lett., 527(2):100-4, 2012.
Ornoy, A. Valproic acid in pregnancy: how much are we endangering the embryo and fetus? Reprod. Toxicol., 28(1):1-10, 2009.

Rajeshwari, T.; Raja, B.; Manivannan, J.; Silambarasan, T. \& Dhanalakshmi, T. Valproic acid prevents the deregulation of lipid metabolism and renal renin-angiotensin system in L-NAME induced nitric oxide deficient hypertensive rats. Environ. Toxicol. Pharmacol., 37(3):936-45, 2014.

Sabers, A.; Bertelsen, F. C.; Scheel-Krüger, J.; Nyengaard, J. \& Møller, A. Long-term valproic acid exposure increases the number of neocortical neurons in the developing rat brain. A possible new animal model of autism. Neurosci. Lett., 580:12-6, 2014.

Sha, K. \& Winn, L. Characterization of valproic acid-initiated homologous recombination. Birth Defects Res. B Dev. Reprod. Toxicol., 89(2):124-32, 2010.

Siéssere, S.; Semprini, M.; Lopes, R.; Sala, M. \& Mattos, M. Morphological and morphometric alterations induced by valproic acid on rat fetuses' meckel's cartilage, lingual musculature, and submandibular gland. Int. J. Morphol., 22(2):133-7, 2004.

Tsai, L. K.; Tsai, M. S.; Ting, C. H. \& Li, H. Multiple therapeutic effects of valproic acid in spinal muscular atrophy model mice. J. Mol. Med. (Berl.), 86(11):1243-54, 2008.

Wang, L.; Liu, Y.; Li, S.; Long, Z. Y. \& Wu, Y. M. Wnt signaling pathway participates in valproic acid-induced neuronal differentiation of neural stem cells. Int. J. Clin. Exp. Pathol., 8(1):578-85, 2015.

Yanamala, N.; Kapralov, A. A.; Djukic, M.; Peterson, J.; Mao, G.; KleinSeetharaman, J.; Stoyanovsky, D. A.; Stursa, J.; Neuzil, J. \& Kagan, V. E. Structural re-arrangement and peroxidase activation of cytochrome c by anionic analogues of vitamin $\mathrm{E}$, tocopherol succinate and tocopherol phosphate. J. Biol. Chem., 289(47):3248898, 2014.

Zhang, B.; Wang, X. \& Nazarali, A. J. Ascorbic acid reverses valproic acid-induced inhibition of hoxa2 and maintains glutathione homeostasis in mouse embryos in culture. Cell. Mol. Neurobiol., 30(1):137-48, 2010.

Dirección para correspondencia:

Dra. Mariana Rojas R.

Laboratorio de Embriología Comparada

Programa de Anatomía y Biología del Desarrollo

Facultad de Medicina, ICBM

Universidad de Chile

Santiago

CHILE

Email: dramrojas@hotmail.com

Recibido : 03-02-2016

Aceptado: 05-04-2016 